\title{
Investigation of the Space Charge Effect in the Quantum Well Infrared Photodetector
}

\author{
C. H. Kuan, Y. F. Hsu, and M. C. Hsu \\ Department of Electrical Engineering \\ National Taiwan University \\ Taipei, Taiwan, R. O. C.
}

\begin{abstract}
The I-V and noise in quantum well infrared photodetectors are investigated. It's found two regimes are in sequence with $V$ and $V^{2}$ dependence of the current. The noise power for the second regime shows $l^{3 / 2}$ dependence. Based on barrier space charge, the first regime is attributed to the transport of residual electrons in equilibrium. The second regime indicates that electron drift velocity and concentration are both proportional to applied biases. All the results is explained with partial potential drop on wells to increase the Fermi level.
\end{abstract}

\section{Introduction}

Recently, there has been much interest in GaAs/AlGaAs quantum well infrared photodetectors (QWIP) in the $10-\mu \mathrm{m}$ range [1-4]. The transport mechanisms in the dark current have been investigated extensively. For the QWIP with thin barriers $(\sim 20 \mathrm{~nm})$, the sequential resonant tunneling dominates through the low and high field domains [5]. For wide barrier $(\sim 30 \mathrm{~nm})$ cases, thermionic emission dominates at low biases while the thermally assisted tunneling plays a major role at high biases $[6,7]$.

In order to cut down the dark current of the QWIP, one of the interesting subjects is to understand the detailed mechanics of the thermionic emission current. In this paper, we report that for the QWIP with 50-nm barriers, the thermionic emission current can be divided into two different regimes in sequence with $V$ and $V^{2}$ dependence of the current. It indicates that the electron emission rate of the thermionic emission current increases with temperatures and biases. A theoretical model based on the electric field produced by the space charge in the wells and barriers is giver to explain the results. The voltage dependence of the current is due to the increment of both of the Fermi level relative to the well edge and the electron concentration in the barrier with the applied bias.

\section{Experiments}

\section{A. Sample Structure}

The investigated QWIP is composed of 31 periods of 5-nm
GaAs wells and $50-\mathrm{nm} \mathrm{Al}_{0.25} \mathrm{Ga}_{0.75} \mathrm{As}$ barriers, and $\mathrm{GaAs}$ contact layers at both ends. The n-type doping density in the wells and contacts is $1.2 \times 10^{18} \mathrm{~cm}^{-3}$. It is noted that the barrier width is thicker than the conventional one of $30 \mathrm{~nm}$. The idea is that the thermally assisted tunneling current is primarily determined by the applied electric field and hence a thicker barrier width may enlarge the initiation bias voltage of the tunneling current. It is then easy to observe the other current components at lower biases.

\section{B. I-V Characteristics}

Fig. 1 shows the full-log I-V curves of the QWIP at different temperatures. It is apparent that there are three different regimes at temperatures between $61 \mathrm{~K}$ and $81 \mathrm{~K}$. The numbers in the figure represent the corresponding slope values. The first regime indicates $I$ is approximately proportional to $V$. For the second regime, the slopes are 2.0 at lower temperatures and 1.7 at $81 \mathrm{~K}$. It implies that $I$ is approximately proportional to $V^{2}$.

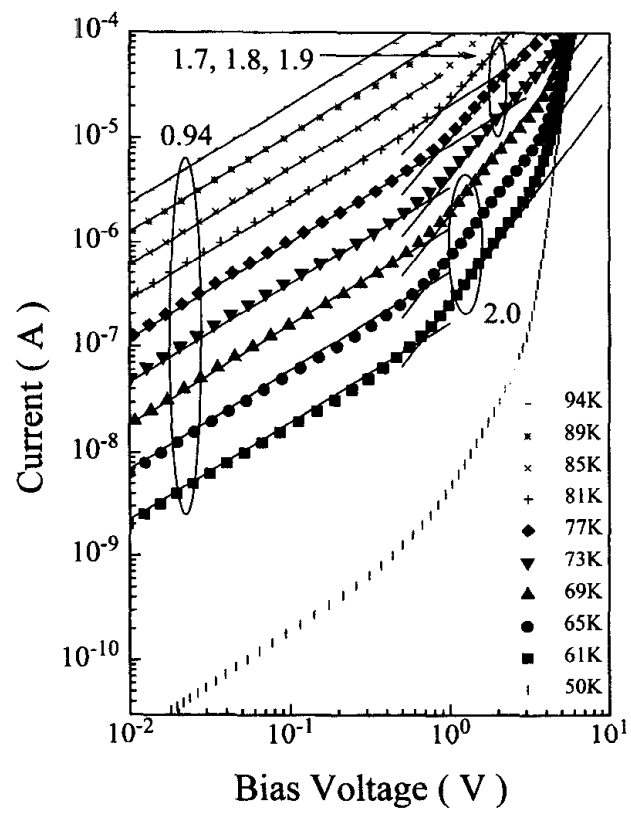

Fig. $1 \mathrm{I}-\mathrm{V}$ curves of the QWIP at different temperatures. It is noted that the current is divided into three different regimes between $61 \mathrm{~K}$ and $81 \mathrm{~K}$. 
The transition biases between the two regimes are found from the intersections of the two relationships. They are shown as the solid circles in the Fig. 5 and will be discussed later. At $50 \mathrm{~K}$, the seperation between the two regimes disappear. In the third regime, the exponential increment of the current is initiated at a fixed bias $3.5 \mathrm{~V}$. It is attributed to the thermally assisted tunneling and indicates the bias profile is independent of temperatures. We are interested to further investigate the first two regimes.

\section{Noise Performance}

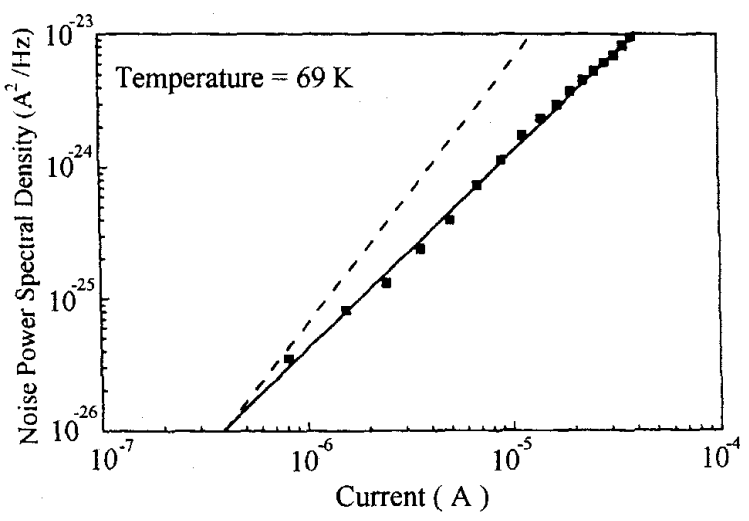

Figure 2 Noise PSD versus current at $69 \mathrm{~K}$. The dashed line represents the associated noise PSD of the photoconductor. The solid squares are the data and the associated slope is $3 / 2$.

Because the barrier may be considered as a resistor, the current through it is proportional to the product of the carrier concentration and drift velocity. To identify their bias dependence, we measure the noise characteristics of the current in the second regime at $69 \mathrm{~K}$. The results are shown as the solid squares in Fig. 2. The noise power spectral density (PSD) follows the $I^{3 / 2}$ relationship as indicated by the solid line. The dashed line represents the general noise relationship of the photoconductor. The deviation indicates the different characteristics of the QWIP and the general photoconductor.

The noise performance of the QWIP can also be described by the generation and recombination noise and the associated PSD is written as

$$
\Delta i^{2}=4 e g I=4 e\left(-\mu_{B} \varepsilon \tau_{f} / L\right) I
$$

where $\mu_{B}$ is the electron mobility in the barrier, $L$ the total length of the QWIP, $\varepsilon$ the electrical field in the barrier, and $\tau_{f}$ the electron lifetime [8]. The noise current gain is denoted by $g$ and equal to the term in the parentheses. Here we have supposed the electron drift velocity is proportional to the electrical field. Since $I$ is proportional to $V^{2}$ and $\varepsilon$ is approximately equal to $-V /(N d)$ where $N$ is the period number, and $d$ the barrier width, $g$ is hence proportional to $I^{1 / 2}$ in this regime and the PSD follows $I^{3 / 2}$ relationship. Therefore the noise characteristics agree with the $I \propto V^{2}$ relationship and $V$ dependence of the drift velocity. It can also be concluded here that the carrier concentration is proportional to $V$ in the second regime of Fig. 1.

\section{Analysis and Discussion}

\section{A. Theoretical Model}

In order to explain the results, we have derived a theoretical model based on the quasi-Fermi level in the barrier. The detailed derivation will be published in another paper, and here the important physical concepts and formulas are given [9].

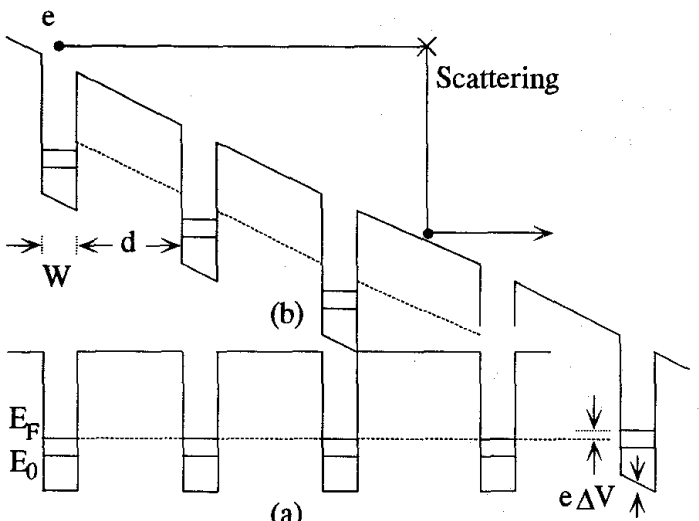

Fig. 3 Band diagrams of the QWIP under (a) thermal equilibrium, and (b) applied biases. In (b), the Fermi level in the barrier as indicated by the dashed line is higher than that in the well because of the scattered electrons coming from the preceding wells.

As shown in Fig. 3, the Fermi level $E_{F}$ is flat at zero bias. The barrier electron concentration $n_{B}$ is given by $n_{t h}=N_{C} \exp \left[\left(E_{B^{-}}\right.\right.$ $\left.\left.E_{F}\right) / k T\right]$ where $N_{C}$ is the effective density of states in the barriers, and $E_{B}$ the barrier height. Under the external bias, the $E_{F}$ relative to the well edge is increased with $e \Delta V$ and the $n_{B}$ is increased with $\Delta n=n_{t h}[\exp (e \Delta V / k T)-1]$. However, because of no doping in the barrier, the mean free path of the electrons is expected to be larger than the $d$. The increment of $n_{B}$ may also result from the electrons generated by the neighboring wells with higher potential energies as shown in Fig. 3. Suppose the total increment is $\gamma \Delta n$ (i.e., $n_{B}=n_{t h}+\gamma \Delta n$ ) where $\gamma$ is a fitting parameter to be extracted from the experimental data. If $V_{B}$ is the potential drop on a single barrier, then the total current is $I=n_{B} e \mu_{B}\left(V_{B} / d\right) A$ where $A$ is the cross sectional area of the QWIP.

To find out $e \Delta V$, the electric field distributions have to be solved in advance. If the $n_{B}$ is small enough, the distribution is uniform and the quasi-Fermi level is a constant relative to the 
conduction band edge even under biases. The electric field produced by the electron charge is hence derived. On the other hand, the wells are heavily doped and degenerate. The electrons can be regarded as three-dimensionally free carriers, or unmovable ones along the growth direction. The former well looks like a conductor while the latter does like an insulator. For these two cases, it is possible to find analytic solutions of the electric field in the well. Besides, by calculating the wave function of the self-consistent ground state of the well under external biases, the numerical result of the electric field can also be found. We will compare the results of these three cases.

For the three-dimensional free carriers and with the center of the well as the origin, the above mensioned procedure gives the potential energy $U(x)$ in the well

$$
U(x)=-\frac{\epsilon_{B}}{\epsilon_{W}} e V_{B} \frac{L_{D}}{d} \sinh \left(\frac{x}{L_{D}}\right) / \cosh \left(\frac{W}{2 L_{D}}\right)
$$

where $\epsilon_{B}$ and $\epsilon_{W}$ are the dielectric constants of the barrier and well respectively, and $W$ the well width. The Debye length is $L_{D}=\sqrt{C_{\gamma} k T \epsilon_{W} /\left(e^{2} N_{D}\right)}$ where $C_{\gamma}$ is equal to $2 F_{1 / 2}(\xi) / F_{-1 / 2}(\xi), \xi=\left(E_{F}-E_{C}\right) / k T$, and $E_{C}$ the conduction band edge. The function $F_{n}(x)$ is the Fermi-Dirac integral with the subscript $n$, i.e., $F_{n}(x)=\int_{0}^{\infty} y^{n} d y /(1+\exp (y-x))$. The calculated Debye length changes from $4.99 \mathrm{~nm}$ at $50 \mathrm{~K}$ to 5.07 $\mathrm{nm}$ at $110 \mathrm{~K}$ and is almost temperature independent. It is noted that because $L_{D}$ and $W$ are compatible, the screening effect is not strong and the potential energy profile in the well is similar to a triangle as that shown in Fig. 3.

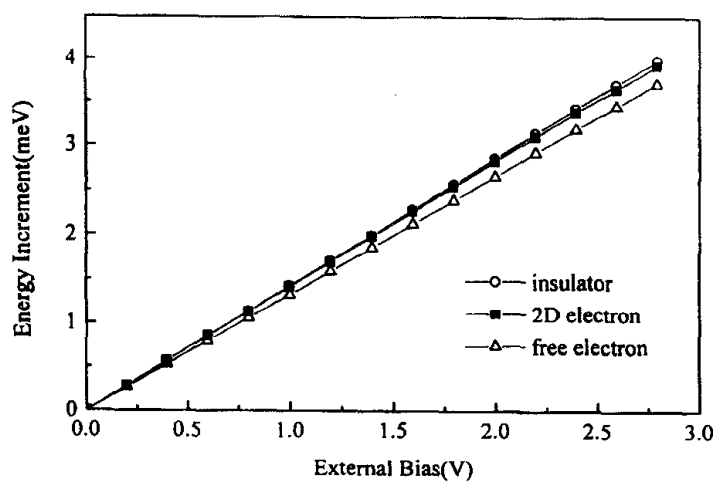

Fig. 4 Fermi energy increment versus the external bias. There are three different cases. The case for the $2 \mathrm{D}$ electrons belong to the self-consistent numerical calculation result. The other two are analytic solutions.

If we regard the well as an insulator, the potential energy profile is $-\left(e V_{B} \in_{B} x\right) /\left(\epsilon_{W} d\right)$. Fig. 4 shows the calculation results of the Fermi energy increment $e \Delta V$ by using the formulas for the insulator and free electrons for $V_{B}=0$ to $3 \mathrm{~V}$. We also utilize the transfer matrix technique and the Poisson equation to calculate the self-consistent solution of the ground state in the well under the external applied bias. The positive ions for the donors in the well are assumed uniformly distributed. The $e \Delta V$ is extracted from the electric field produced by the ions and electrons. The differences among them are less than $8 \%$ at $V_{B}=2.75 \mathrm{~V}$. Therefore, for the electrons in the well, $e \Delta V$ can be estimated by (2) and is equal to $-U(W / 2)$. From the same equation, the relationship between $V_{\mathrm{B}}$ and total applied bias $V$ can also be found,

$$
V=\left[\left(1+2 \frac{\epsilon_{B}}{\epsilon_{W}} \frac{L_{D}}{d} \tanh \left(\frac{W}{2 L_{D}}\right)\right) N\right] V_{B}=C_{T} V_{B}
$$

where $C_{T}$ is a transform constant and equal to the term in the bracket.

Due to the increment of $E_{F}$, and the assumption $e \Delta V<<k T$, the current $I$ may be written as

$$
I=n_{B} e \mu_{B}\left(\frac{V_{B}}{d}\right) A=\left(1+\frac{V}{V_{t r}}\right) n_{t h} e \mu_{B} \frac{V}{C_{T} d} A
$$

where $V_{t r}$ is the transition bias

$$
V_{t r}=\frac{1}{\gamma}\left(\frac{\epsilon_{W}}{\epsilon_{B}} \frac{N k T}{e} \frac{d}{L_{D}} \operatorname{coth}\left(\frac{W}{2 L_{D}}\right)+2 \frac{N k T}{e}\right)
$$

It is apparent that (4) can explain the two regimes found in Fig. 1. Besides, (3) and the almost independence of the Debye length on the temperature give a good reason why the thermally assisted tunneling current is initiated at a fixed bias at different temperatures.

\section{B. Comparison with Experiments}

Utilizing $\gamma$ in (5) as a parameter to fit the solid circles in Fig. 5 , we find that $\gamma$ is equal to 6.1 and the result is shown as the solid line. The $\gamma$ value indicates that the injected electrons from the preceding sixth well can contribute to the carrier concentration in the barrier. With (4) and I-V curves, the $\mu_{B}$ at different temperatures can be estimated and the results are shown as the solid triangles in Fig. 5. The dashed curve represents the mobility to follow the $T^{3 / 2}$ dependence due to the scattering of the phonons. The measured electron Hall mobility at $77 \mathrm{~K}$ for pure n-type GaAs sample is about $2 \times 10^{5}$ $\mathrm{cm}^{2} / \mathrm{V}$-s [10]. Our data show that the electron mobility in $\mathrm{Al}_{0.25} \mathrm{Ga}_{0.75} \mathrm{As}$ barriers is about $10^{4} \mathrm{~cm}^{2} / \mathrm{V}$-s at the same temperature, which is a reasonable value [11]. 
At $50 \mathrm{~K}$, the I-V curve does not have an apparent $V^{2}$ dependent regime. It may be explained with (2). Taking $V_{B} \approx$ $1.0 / 31 \mathrm{~V}, T=50 \mathrm{~K}$ and $\epsilon_{B} / \epsilon_{W}=12.4 / 13.18$, (2) gives $e \Delta V=$ $1.4 \mathrm{meV}$, which is equal to $0.33 k T$. The approximation of $\exp (e \Delta V / k T)$ with $(1+e \Delta V / k T)$ is not appropriate for higher biases. Therefore, the data show higher than $V^{2}$ dependence under the biases around 1 to $3.5 \mathrm{~V}$. From Fig. 2, the ratio of the noise power spectral density over $I^{3 / 2}$ can be found. Combining (1) and (4) with the ratio, we find that the electron lifetime is $0.56 \mathrm{ps}$ for our QWIP, which is close to the result measured by subpicosecond luminescence at $69 \mathrm{~K}$ [12].

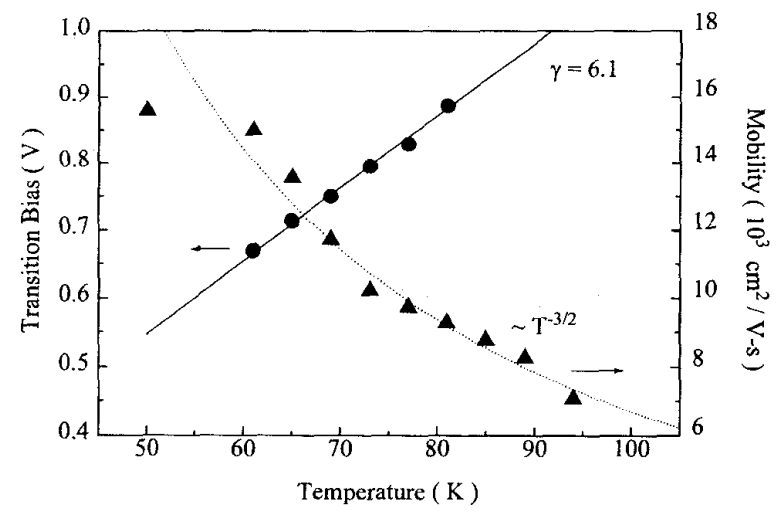

Fig. 5 Transition bias and mobility versus temperature. The solid circles represent the experimental results of transition biases while the solid line is the theoretical values. The mobility as represented by solid triangles is derived from the conductance of the sample at low biases. The dashed curve shows temperature dependence of the mobility due to scattering of phonons.

The similar I-V characteristics also appear in the measurement of the resistivity of semi-insulating epitaxial layer [13]. The $V$ dependent regime is explained with the thermal equilibrium electron concentration while $V^{2}$ dependent one is with the high injection of carriers from the contact. In our QWIP case, the high injection is due to the increment of the Fermi level relative to the well edge by the applied bias.

\section{Summary}

In summary, the I-V characteristics and noise performance before electron tunneling in our QWIP show the electron concentration in the barrier increases with bias and temperature. It can be explained with our theoretical model in which the increment of the Fermi level with the applied bias renders the increment of the concentration.

This work was supported by the National Science Council of the Republic of China under contract number NSC 872215-E-002-005.

\section{Reference}

[1] L. C. West and S. J. Eglash, "First observation of an extremely largedipole infrared transition within the conduction band of a GaAs quantum well," Appl. Phys. Lett. Vol. 46, pp. 1156-1158, June 1985.

[2] B. F. Levine, C. G. Bethea, G. Hasnain, V. O. Shen, E. Pelve, R. R Abbott, and S. J. Hsieh, "High sensitivity low dark current $10 \mu \mathrm{m} \mathrm{GaAs}$ quantum well infrared photodetectors," Appl. Phys. Lett. Vol. 56, pp. 851-853, Febrary 1990

[3] J. Y. Andersson and L. Lundquivst, "Near-unity quantum efficiency of $\mathrm{AlGaAs} / \mathrm{GaAs}$ quantum well infrared detectors using a waceguide with a doubly periodic granting coupler," Appl. Phys. Lett. Vol. 59, pp. 857859, June 1991.

[4] L. S. Yu and S. S. Li, "A metal grating coupled bound-to-miniband transition GaAs multiquantum well/superlattice infrared detector," Appl. Phys. Lett. Vol. 59, pp. 1332-1334 September 1991.

[5] K. K. Choi, B. F. Levine, R. J. Malik, J. Walker, and C. G. Bethea, "Periodic negative conductance by sequential resonant tunneling through an expanding high-field superlattice domain," Phys. Rev. B Vol. 35, pp. 4172-4175, March 1987.

[6] E. Pelve, F. Beltram, C. G. Bethea, B. F. Levine, V. O. Shen, S. J. Hsieh, and R. R. Abbott, "Analysis of the dark current in doped-well multiple quantum well AlGaAs infrared photodetectors," J. Appl. Phys. Vol. 66, pp. 5656-5658, December 1989.

[7] C. H. Kuan, D. C. Tsui, K. K. Choi, P. G. Newman, and W. H. Chang, "Hot-electron distribution in multiple quantum well infrared photodetectors," Appl. Phys. Lett. Vol. 63, pp. 2091-2093, October 1993.

[8] A. Rose, Concepts in Photoconductivity and Applied Problems (Interscience, New York, 1963).

[9] C. H. Kuan and C. C. Chen. "Electron Transport Mechanism Before Tunneling in the Quantum Well Infrared Photodetector" (unpublished)

[10] J. S. Blakemore, "Semiconducting and other major properties of gallium arsenide," J. Appl. Phys., Vol. 53, pp. 123-181, 1982.

[11] W. C. Liu, "Investigation of electrical and photoluminescent properties of MBE grown $\mathrm{Al}_{\mathrm{x}} \mathrm{Ga}_{1-\mathrm{x}} \mathrm{As}$ layers," J. Materials Science, Vol. 25, pp. 1765-1772, March 1990

[12] B. Deveaud, A. Chomette, F. Clerot, and Regreny, Intersubband Transitions in Quantum Well, edited by Emmanuel Rosencher etc., Plenum Press, New York, 1992.

[13] V. Swaminathan and A. T. Macrander, Materials Aspects of GaAs and InP Based Structure, pp. 255-261 (Prentica Hall, New Jersey, 1991). 